\title{
Sciscionella marina gen. nov., sp. nov., a marine actinomycete isolated from a sediment in the northern South China Sea
}

\author{
Correspondence \\ Wen-Jun Li \\ wjli@ynu.edu.cn \\ Si Zhang \\ zhangsi@scsio.ac.cn
}

\author{
Xin-Peng Tian, ${ }^{1,2} \dagger$ Xiao-Yang Zhi, ${ }^{2} \dagger$ Yun-Qi Qiu, ${ }^{1}$ Yu-Qin Zhang, ${ }^{2}$ \\ Shu-Kun Tang, ${ }^{2}$ Li-Hua Xu, ${ }^{2}$ Si Zhang ${ }^{1,3}$ and Wen-Jun $\mathrm{Li}^{1,2}$
}

${ }^{1}$ Guangdong Key Laboratory of Marine Materia Medica, South China Sea Institute of Oceanology, Chinese Academy of Sciences, Guangzhou, 510301, PR China
${ }^{2}$ The Key Laboratory for Microbial Resources of the Ministry of Education, PR China, and Laboratory for Conservation and Utilization of Bio-resources, Yunnan Institute of Microbiology, Yunnan University, Kunming, Yunnan, 650091, PR China
${ }^{3}$ Hainan Key Lab of Tropical Marine Biotechnology, South China Sea Institute of Oceanology, Chinese Academy of Sciences, Sanya, 572000, PR China

More than $70 \%$ of our planet's surface is covered by oceans, which play a crucial role in the global ecological system. Unexplored marine environments are now a popular research area due to the potentially huge resources present within them. Recently, marine actinomycete research has received more attention, especially after the establishment of the new genus Salinispora and the discovery that it is an excellent source of secondary metabolites (Laatsch, 2006; Lam, 2006). Many novel bioactive secondary metabolites isolated from marine actinomycetes have been reported (Lam, 2006), and they may be a source of novel compounds with pharmaceutical potential (Fiedler et al., 2005; Jensen et al., 2005a; Fenical \& Jensen, 2006).

†These authors contributed equally to this work.

The GenBank/EMBL/DDBJ accession number for the 16S rRNA gene sequence of strain SCSIO $00231^{\top}$ is EU503139.

Scanning electron micrographs of mycelium of strain SCSIO $00231^{\top}$ are available as supplementary material with the online version of this paper.
Culture-independent studies have shown that marine sediment environments contain a wide diversity of actinomycetes, and many unique taxa are very different from their terrestrial counterparts (Stach et al., 2003; Gontang et al., 2007). In addition, culture-dependent studies have also shown that marine actinomycetes are ubiquitous in marine sediment environments (Maldonado et al., 2005; Jensen et al., 2005b; Gontang et al., 2007). During an investigation of the diversity of cultivable marine actinomycetes, strain SCSIO $00231^{\mathrm{T}}$ was isolated from a grey sand sediment sample. Based on phylogenetic analysis, morphological and physiological data and chemotaxonomic markers, strain SCSIO $00231^{\mathrm{T}}$ can be readily distinguished from described genera and represents a new member of the family Pseudonocardiaceae. Here, we report the taxonomic description of this strain.

Samples were collected in September 2006 from the northern South China Sea $\left(20^{\circ} 36^{\prime} \mathrm{N} 116^{\circ} 21^{\prime} \mathrm{E}\right.$; depth $516 \mathrm{~m}$ ). The surface layer of the sediment, about $40 \mathrm{~cm}$ in 
depth, was collected by a grab bucket, and the top $10 \mathrm{~cm}$ layer was obtained aseptically for sampling; samples were placed in sterile $50 \mathrm{ml}$ conical tubes. All samples were processed for cultivation experiments by using a standard dilution plating method on ship within $2 \mathrm{~h}$, and the remainder was frozen at $-20{ }^{\circ} \mathrm{C}$. Strain SCSIO $00231^{\mathrm{T}}$ was isolated on Gauze No. 1 medium prepared with seawater instead of distilled water, incubated at $28{ }^{\circ} \mathrm{C}$ for 3 weeks. The purified strain was maintained on modified ISP medium 2 (prepared with natural seawater) (Shirling \& Gottlieb, 1966) and as $20 \%$ (w/v) glycerol suspensions at $-20{ }^{\circ} \mathrm{C}$. Biomass for chemotaxonomic and molecular systematic studies was obtained by cultivation using modified ISP 2 broth $\left(28{ }^{\circ} \mathrm{C}\right.$, 1 week, 150 r.p.m.).

Strain SCSIO $00231^{\mathrm{T}}$ grew well on media ISP 2, ISP 4 and ISP 5 (Shirling \& Gottlieb, 1966), Czapek solution agar (Waksman, 1961), nutrient agar (Difco) and potato agar (Waksman, 1961), but not on ISP 3 medium at $28{ }^{\circ} \mathrm{C}$. Diffusible pigments were not observed and the colony colour was yellow-white, examined by comparing the cultures with the most suitable colour chips from the ISCC-NBS Color Charts (Kelly, 1964). Micromorphology was examined by light microscopy (model BH 2; Olympus) and electron microscopy (JSM5600LV; JEOL) using cells incubated in modified ISP 2 medium for 14 and 28 days. The organism formed branching substrate mycelium and fragmented into rod-shaped elements, 2.5-3.5 $\mu \mathrm{m}$ long. Sparse mycelium was produced on modified ISP 2 medium after incubation for 28 days (Supplementary Fig. S1, available in IJSEM Online).

The growth temperature was tested at $4-55{ }^{\circ} \mathrm{C}$ and $\mathrm{pH}$ range for growth was determined at $\mathrm{pH} 4.0-12.0$, based on the buffer system described by Xu et al. (2005), using modified ISP 2 as the basal medium. Tolerance of $\mathrm{NaCl}$ was examined at $0-20 \%(\mathrm{w} / \mathrm{v})$. Carbon source utilization $(0.5 \%, \mathrm{w} / \mathrm{v})$ was tested as described by Shirling \& Gottlieb (1966). Physiological tests including hydrolysis of cellulose, gelatin, starch and Tweens 20,40,60 and 80, nitrate reduction, utilization of urea, milk coagulation and peptonization and $\mathrm{H}_{2} \mathrm{~S}$ and melanin production were performed as described previously (Gonzalez et al., 1978; Smibert \& Krieg, 1981). Antibiotic susceptibility was examined as described by Groth et al. (2004) using antibiotic discs on modified ISP 2 medium.

Cells of strain SCSIO $00231^{\mathrm{T}}$ were Gram-positive and aerobic. The strain was susceptible to ( $\mu \mathrm{g}$ per disc) penicillin $G(10)$, erythromycin (15), gentamicin (10), novobiocin (30), trimethoprim (1.25), netilmicin (30), amikacin (30), tobramycin (10) and neomycin (10) and resistant to streptomycin (10), tetracycline (30), vancomycin (30), lincomycin (2), rifampicin (5), chloramphenicol (30), ampicillin (10), norfloxacin (10), amoxicillin (10) and ciprofloxacin (5). Detailed physiological properties of the strain are given in the species description.

Analysis of whole-cell sugars was done according to procedures described by Staneck \& Roberts (1974).
Amino acids and peptides in cell-wall hydrolysates were analysed by the methods described by Schleifer (1985) and Schleifer \& Kandler (1972) with the modification that TLC on cellulose sheets was applied instead of paper chromatography. Menaquinones were isolated using the methods of Minnikin et al. (1984) and separated by HPLC (Kroppenstedt, 1982; Kroppenstedt et al., 1981). Phospholipids were extracted and examined by using published procedures (Minnikin $e t$ al., 1979; Collins \& Jones, 1980). Fatty acid analysis was performed by using standard methods (Sasser, 1990) and the results were compared with the database of fatty acids in the Microbial Identification System.

The isolate had a type IV cell wall; whole-cell hydrolysates contained meso-diaminopimelic acid and the whole-cell sugars were galactose, arabinose and glucose. Phospholipids were type III, including diphosphatidylglycerol, phosphatidylmethylethanolamine, phosphatidylethanolamine, phosphatidylcholine, phosphatidylinositol and unknown phosphoglycolipids. The predominant menaquinone was MK-9 $\left(\mathrm{H}_{4}\right)(93 \%)$. The $\mathrm{G}+\mathrm{C}$ content of the genomic DNA was $69 \mathrm{~mol} \%$, determined by using the HPLC method (Mesbah et al., 1989). The fatty acid profile contained $\mathrm{i}-\mathrm{C}_{16: 0}(43.9 \%)$, unknown fatty acid (peak name 16.048$)(15.52 \%), \mathrm{i}-\mathrm{C}_{16: 0} 2-\mathrm{OH}(12.70 \%)$, ai- $\mathrm{C}_{17: 0}$ (9.84\%), ai- $\mathrm{C}_{17: 0} \quad 2-\mathrm{OH} \quad(3.02 \%), \quad 10$-methyl $\mathrm{C}_{16: 0}$ $(2.81 \%), \quad$ i-C $\mathrm{C}_{15: 0} \quad(2.48 \%), \quad \mathrm{i}-\mathrm{C}_{17: 0} \quad(2.21 \%), \quad \mathrm{C}_{16: 0}$ $(1.87 \%), \mathrm{C}_{14: 0}(1.82 \%)$, ai- $\mathrm{C}_{15: 0}(1.08 \%), \mathrm{C}_{18: 0} 3-\mathrm{OH}$ $(0.89 \%), \quad \mathrm{i}-\mathrm{C}_{15: 0} \quad 2-\mathrm{OH} / \mathrm{C}_{16: 1}$ t9 $(0.73 \%), \mathrm{C}_{16: 1} 2-\mathrm{OH}$ $(0.56 \%)$ and $\mathrm{i}-\mathrm{C}_{14: 0}(0.55 \%)$. Chemotaxonomic characteristics of strain SCSIO $00231^{\mathrm{T}}$ and its closest phylogenetic neighbours are compared in Table 1 .

Extraction of genomic DNA and PCR amplification of the $16 \mathrm{~S}$ rRNA gene were done as described by Li et al. (2007). Multiple alignments with sequences of the most closely related taxa and calculations of levels of sequence similarity were carried out using CLUSTAL_X (Thompson et al., 1997). A phylogenetic tree and distance matrix were reconstructed using the neighbour-joining method of Saitou \& Nei (1987) from $K_{\text {nuc }}$ values (Kimura, 1980, 1983) using MEGA version 4.0 (Tamura et al., 2007). The topology of the phylogenetic tree was evaluated by the bootstrap resampling method of Felsenstein (1985) with 1000 replicates.

BLAST search results using the 16S rRNA gene sequence of strain SCSIO $00231^{\mathrm{T}}$ showed that the new isolate had the highest similarities to members of the family Pseudonocardiaceae, such as Saccharopolyspora erythraea NRRL $2338^{\mathrm{T}}$ (92.6\% similarity), Thermocrispum municipale DSM $44069^{\mathrm{T}}(93 \%)$ and Amycolatopsis sacchari DSM $44468^{\mathrm{T}}(93.1 \%)$. Additionally, patterns of selected $16 \mathrm{~S}$ rRNA gene signature nucleotides defined for the family Pseudonocardiaceae (Stackebrandt et al., 1997) were also consistent with nucleotides determined for the 16S rRNA gene sequence of strain SCSIO $00231^{\mathrm{T}}$, except that $\mathrm{G}-\mathrm{G}$ and $U$ were determined at positions $183: 194$ and 747 , respectively. All the above data confirmed that the new isolate should be assigned to the family Pseudonocardiaceae. 
Table 1. Chemotaxonomic characteristics of strain SCSIO $00231^{\top}$ and its closest relatives in described genera of the family Pseudonocardiaceae

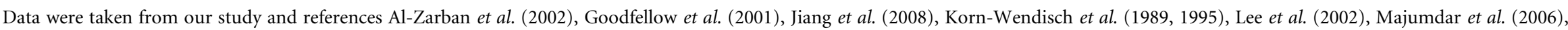
Mertz \& Yao (1993) and Tomita et al. (1993). Cell walls of all taxa contain meso-diaminopimelic acid.

\begin{tabular}{|c|c|c|c|c|c|c|}
\hline Strain & $\begin{array}{l}\text { Cell-wall } \\
\text { type }\end{array}$ & $\begin{array}{l}\text { Whole-cell } \\
\text { sugars }^{*}\end{array}$ & $\begin{array}{l}\text { Phospholipid } \\
\text { type }\end{array}$ & Phospholipids $\dagger$ & $\begin{array}{l}\text { Predominant } \\
\text { menaquinone }\end{array}$ & Major fatty acid(s) (>10\%) \\
\hline Strain SCSIO $00231^{\mathrm{T}}$ & IV & Ara, Gal, Glc & P III & DPG, PC, PE, PI, PL, PME & $9\left(\mathrm{H}_{4}\right)$ & $\mathrm{i}-\mathrm{C}_{16: 0}, \mathrm{i}-\mathrm{C}_{16: 0} 2-\mathrm{OH}$ \\
\hline Thermocrispum municipale DSM $44069^{\mathrm{T}}$ & III & Ara, Man, Glc & P II & PE, PE-OH, PI & $9\left(\mathrm{H}_{4}\right)$ & $\mathrm{i}-\mathrm{C}_{16: 0}$ \\
\hline Amycolatopsis orientalis NBRC $12806^{\mathrm{T}}$ & IV & Ara, Gal & P II & DPG, PE, PG, PI, PME & $9\left(\mathrm{H}_{4}\right)$ & $\begin{array}{l}\mathrm{i}-\mathrm{C}_{15: 0}, \mathrm{i}-\mathrm{C}_{16: 0}, \mathrm{C}_{16: 1} \omega 6 c / \mathrm{C}_{16: 1} \omega 7 c \\
\mathrm{C}_{17: 1} \omega 8 c\end{array}$ \\
\hline Amycolatopsis sacchari DSM $44468^{\mathrm{T}}$ & IV & Ara, Gal & P II & DPG, PE, PE-OH, PG, PI & $9\left(\mathrm{H}_{4}\right)$ & $\mathrm{i}-\mathrm{C}_{16: 0}$, ai- $\mathrm{C}_{17: 0}$ \\
\hline Saccharopolyspora hirsuta DSM $43463^{\mathrm{T}}$ & IV & Ara, Gal & P III & $\begin{array}{l}\text { DPG, PC, PE, lyso-PE, PE-OH, } \\
\text { PI, PME }\end{array}$ & $9\left(\mathrm{H}_{4}\right)$ & $\mathrm{i}-\mathrm{C}_{15: 0}, \mathrm{i}-\mathrm{C}_{16: 0}, \mathrm{i}-\mathrm{C}_{17: 0}$, ai- $\mathrm{C}_{17: 0}$ \\
\hline Saccharopolyspora erythraea NRRL $2338^{\mathrm{T}}$ & IV & Ara, Gal & P III & DPG, PC, PE, lyso-PE, PI & $9\left(\mathrm{H}_{4}\right)$ & $\mathrm{i}-\mathrm{C}_{15: 0}, \mathrm{i}-\mathrm{C}_{16: 0}, \mathrm{i}-\mathrm{C}_{17: 0}$, ai- $\mathrm{C}_{17: 0}, \mathrm{C}_{17: 0}$ \\
\hline Prauserella rugosa DSM $43194^{\mathrm{T}}$ & IV & Ara, Gal & P II & DPG, PE, PG, PI, PME & $9\left(\mathrm{H}_{2,4}\right)$ & $\mathrm{i}-\mathrm{C}_{16: 0}, \mathrm{i}-\mathrm{C}_{16: 1} \mathrm{H}, \mathrm{C}_{16: 1}, \mathrm{C}_{17: 1} \mathrm{C}$ \\
\hline Saccharomonospora halophila DSM $44411^{\mathrm{T}}$ & IV & Ara, Gal & P II & DPG, PI, PE PE-OH, lyso-PE & $9\left(\mathrm{H}_{4}\right)$ & $\mathrm{i}-\mathrm{C}_{16: 0}, \mathrm{C}_{16: 0}, \mathrm{C}_{16: 1}, \mathrm{i}-\mathrm{C}_{16: 0} 2-\mathrm{OH}$ \\
\hline Pseudonocardia spinosispora LM $141^{\mathrm{T}}$ & IV & Ara, Gal & P III & $\begin{array}{l}\text { DPG, PC, PE, PE-OH, PG, PIM, } \\
\text { PL, PME }\end{array}$ & $8\left(\mathrm{H}_{4}\right)$ & $\mathrm{i}-\mathrm{C}_{15: 0}, \mathrm{i}-\mathrm{C}_{16: 0}, \mathrm{i}-\mathrm{C}_{17: 0}$ \\
\hline $\begin{array}{l}\text { Kibdelosporangium aridum subsp. largum DSM } \\
44150^{\mathrm{T}}\end{array}$ & IV & Ara, Gal, Glc, Rha & P II & PE, PG, PI, PME & $9\left(\mathrm{H}_{4}\right)$ & $\mathrm{i}-\mathrm{C}_{16: 0}, \mathrm{C}_{16: 0}$, ai- $\mathrm{C}_{17: 0}, \mathrm{C}_{17: 0}$ \\
\hline Actinomycetospora chiangmaiensis YIM $0006^{\mathrm{T}}$ & IV & Ara, Gal & P III & PC, PG, PI & $9\left(\mathrm{H}_{4}\right)$ & $\mathrm{C}_{16: 1} \omega 7 c / \mathrm{i}-\mathrm{C}_{15: 0} 2-\mathrm{OH}, \mathrm{i}-\mathrm{C}_{16: 0}, \mathrm{C}_{16: 0}$ \\
\hline
\end{tabular}

*Ara, Arabinose; Gal, galactose; Glc, glucose; Man, mannose; Rha, rhamnose.

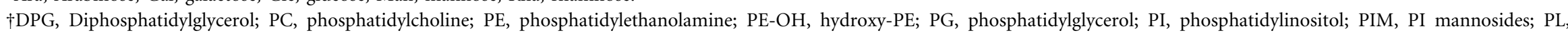
unknown phospholipids; PME, phosphatidylmethylethanolamine. 


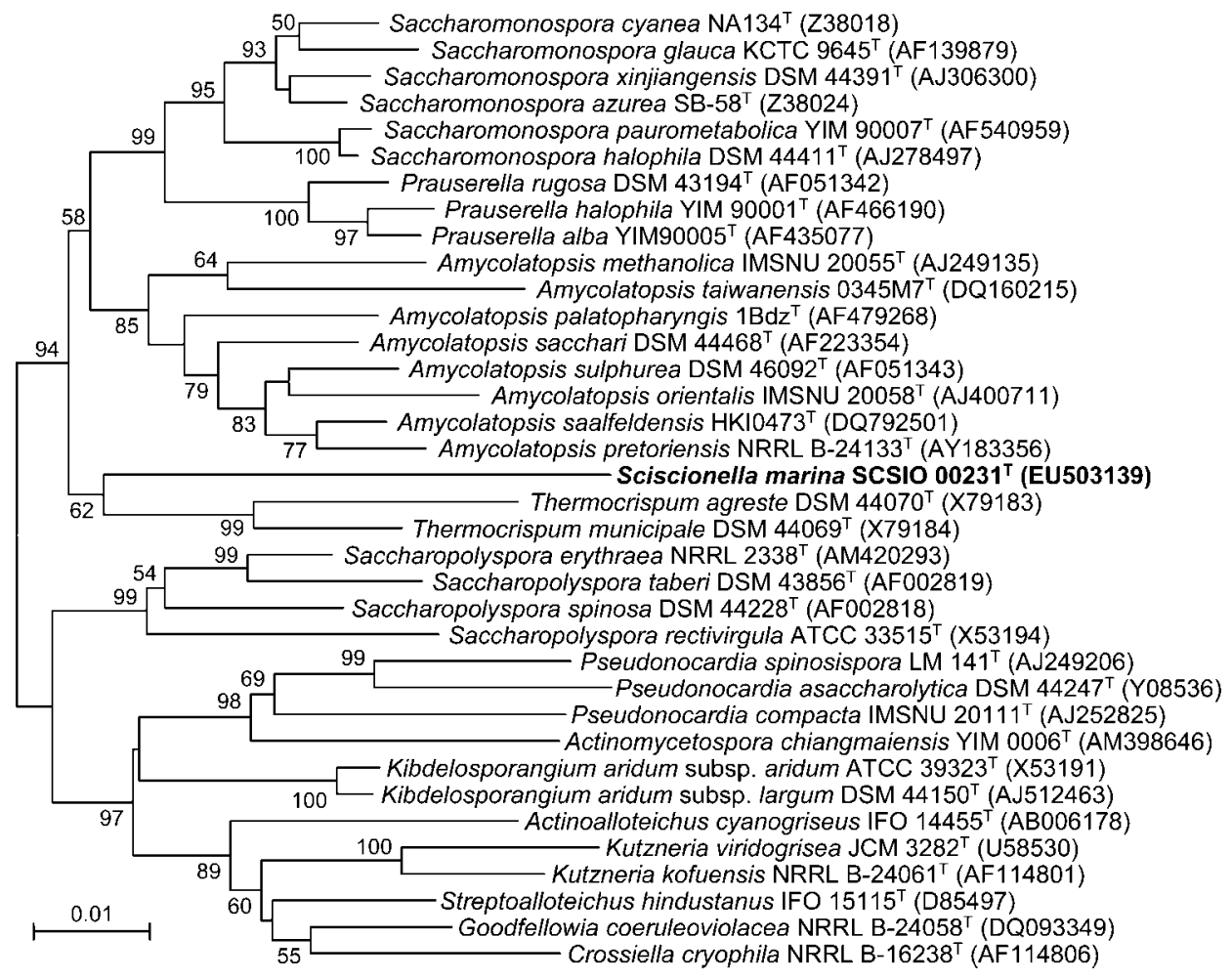

Fig. 1. Phylogenetic dendrogram of strain SCSIO $00231^{\top}$ and its closest relatives within the family Pseudonocardiaceae, reconstructed by using the neighbour-joining method, based on 16S rRNA gene sequences of more than 1300 nt. Numbers at nodes are bootstrap percentages based on 1000 resamplings (only values of $50 \%$ or more are indicated). Bar, $1 \%$ sequence divergence.

In the phylogenetic tree based on the $16 \mathrm{~S}$ rRNA gene sequences of representatives of all genera in family Pseudonocardiaceae (Fig. 1), strain SCSIO $00231^{\mathrm{T}}$ formed a distinct lineage and was loosely associated with the genus Thermocrispum, with the highest similarity of $93 \%$ to Thermocrispum municipale DSM $44069^{\mathrm{T}}$, which showed the earlier evolutionary divergence between strain SCSIO $00231^{\mathrm{T}}$ and the other previously described genera in this family. Chemotaxonomic characteristics could be distinguished readily from related taxa in the family Pseudonocardiaceae (Table 1). We also compared the nucleotide signatures, which also indicate many distinctions between strain SCSIO $00231^{\mathrm{T}}$ and other genera of the family Pseudonocardiaceae in some signature sites (Table 2). Thus, based on the phylogenetic position and chemotaxonomic data, a novel genus is proposed for strain SCSIO $00231^{\mathrm{T}}$, to be named Sciscionella gen. nov., with the type species Sciscionella marina sp. nov.

\section{Description of Sciscionella gen. nov.}

Sciscionella (Sci.sci.o.nel'la. N.L. fem. dim. n. Sciscionella arbitrary name formed from the acronym of the South China Sea Institute of Oceanology, SCISCIO, where taxonomic studies on this taxon were performed).
Gram-positive, aerobic organisms that produce fragmented substrate mycelium and sparse aerial mycelium on modified ISP 2 medium. Characterized by cell-wall chemotype IV, containing meso-diaminopimelic acid and whole-cell sugars arabinose, galactose and glucose, and phospholipid pattern type III sensu Lechevalier et al. (1977), comprising diphosphatidylglycerol, phosphatidylmethylethanolamine, phosphatidylethanolamine, phosphatidylcholine, phosphatidylinositol and unknown phospholipids. Mycolic acids are absent. The predominant menaquinone is $\mathrm{MK}-9\left(\mathrm{H}_{4}\right)$. Major fatty acids are $\mathrm{i}-\mathrm{C}_{16: 0}$, ai- $\mathrm{C}_{17: 0}$ and $\mathrm{i}-\mathrm{C}_{16: 0}$ 2-OH. The $\mathrm{G}+\mathrm{C}$ content of the genomic DNA is about $69 \mathrm{~mol} \%$. The type species is Sciscionella marina.

\section{Description of Sciscionella marina sp. nov.}

Sciscionella marina (ma.ri'na. L. fem. adj. marina of the sea).

Morphological, chemotaxonomic and general characteristics are as given above for the genus. Colonies are yellowwhite on most tested media. Good growth occurs at $28{ }^{\circ} \mathrm{C}$ on media ISP 2, ISP 4, ISP 5, Czapek solution agar, nutrient agar and potato agar. No diffusible pigments are 
Table 2. Patterns of selected 16S rRNA gene signature nucleotides detected among the various genera of the family Pseudonocardiaceae

Taxa: 1, SCSIO $00231^{\mathrm{T}}$; 2, Thermocrispum; 3, Amycolatopsis; 4, Saccharopolyspora; 5, Saccharomonospora; 6, Prauserella; 7, Pseudonocardia; 8, Kutzneria; 9, Actinoalloteichus; 10, Crossiella; 11, Kibdelosporangium; 12, Streptoalloteichus; 13, Goodfellowia; 14, Actinostreptospora; 15, Actinomycetospora. All $16 \mathrm{~S}$ rRNA gene sequences of type strains belong to the family Pseudonocardiaceae were included in this analysis. The signatures given below for each group were chosen for their presence in more than $95 \%$ of the members of the respective genera. -, Residue absent; *, no signature residue.

\begin{tabular}{|c|c|c|c|c|c|c|c|c|c|c|c|c|c|c|c|}
\hline Position(s) & 1 & 2 & 3 & 4 & 5 & 6 & 7 & 8 & 9 & 10 & 11 & 12 & 13 & 14 & 15 \\
\hline $42: 400$ & $\mathrm{U}-\mathrm{C}$ & $\mathrm{G}-\mathrm{C}$ & $\mathrm{G}-\mathrm{C}$ & $\mathrm{G}-\mathrm{C}$ & $\mathrm{G}-\mathrm{C}$ & $\mathrm{G}-\mathrm{C}$ & $\mathrm{G}-\mathrm{C}$ & $\mathrm{G}-\mathrm{C}$ & $\mathrm{G}-\mathrm{C}$ & $\mathrm{G}-\mathrm{C}$ & $\mathrm{G}-\mathrm{C}$ & $\mathrm{G}-\mathrm{C}$ & $\mathrm{G}-\mathrm{C}$ & $\mathrm{G}-\mathrm{C}$ & $\mathrm{G}-\mathrm{C}$ \\
\hline $43: 399$ & U-G & $\mathrm{C}-\mathrm{G}$ & $C-G$ & $\mathrm{C}-\mathrm{G}$ & $\mathrm{C}-\mathrm{G}$ & $\mathrm{C}-\mathrm{G}$ & $\mathrm{C}-\mathrm{G}$ & $\mathrm{C}-\mathrm{G}$ & $\mathrm{C}-\mathrm{G}$ & $\mathrm{C}-\mathrm{G}$ & C-G & $\mathrm{C}-\mathrm{G}$ & $\mathrm{C}-\mathrm{G}$ & C-G & $C-G$ \\
\hline 51 & $\mathrm{U}$ & A & $\mathrm{A} / \mathrm{C}$ & A & A & A & A & A & A & A & A & A & A & A & A \\
\hline $52: 359$ & $A-G$ & C-G & $C-G$ & $\mathrm{C}-\mathrm{G}$ & $\mathrm{C}-\mathrm{G}$ & C-G & $\mathrm{C}-\mathrm{G}$ & C-G & $\mathrm{C}-\mathrm{G}$ & C-G & C-G & $\mathrm{C}-\mathrm{G}$ & C-G & C-G & $C-G$ \\
\hline $70: 98$ & G-G & U-A & $\mathrm{U}-\mathrm{A}$ & $\mathrm{U}-\mathrm{A}$ & U-A & $\mathrm{U}-\mathrm{A}$ & $\mathrm{U}-\mathrm{A}$ & ${ }^{*}-\mathrm{A}$ & $\mathrm{U}-\mathrm{A}$ & U-A & $\mathrm{U}-\mathrm{A}$ & $\mathrm{U}-\mathrm{A}$ & U-A & $\mathrm{U}-\mathrm{A}$ & $\mathrm{U}-\mathrm{A}$ \\
\hline 72 & $\mathrm{C}$ & A & - & - & A & A & - & A & A & A & A & A & A & A & A \\
\hline $183: 194$ & G-G & $\mathrm{G}-\mathrm{C}$ & ${ }^{*}-\mathrm{G}$ & G-G & $\mathrm{G}-\mathrm{C}$ & $\mathrm{G}-\mathrm{C}$ & G-G & $\mathrm{G}-\mathrm{C}$ & $\mathrm{G}-\mathrm{C}$ & $\mathrm{G}-\mathrm{C}$ & $\mathrm{G}-\mathrm{C}$ & $\mathrm{G}-\mathrm{C}$ & $\mathrm{G}-\mathrm{C}$ & $\mathrm{G}-\mathrm{C}$ & $\mathrm{G}-\mathrm{C}$ \\
\hline 208 & G & G & $\mathrm{U}$ & $\mathrm{U}$ & $\mathrm{U}$ & G & $\mathrm{U}$ & G & G & G & G & G & G & G & G \\
\hline 210 & A & A & G & G & G & A & G & A & A & A & A & A & A & A & A \\
\hline 213 & G & G & A & A & A & C & A & G & $\mathrm{U}$ & $\mathrm{U}$ & $\mathrm{U}$ & $\mathrm{U}$ & $\mathrm{U}$ & $\mathrm{U}$ & $\mathrm{U}$ \\
\hline $248: 276$ & U-G & $\mathrm{C}-\mathrm{G}$ & $\mathrm{C}-\mathrm{G}$ & $\mathrm{C}-\mathrm{G}$ & $\mathrm{C}-\mathrm{G}$ & $\mathrm{C}-\mathrm{G}$ & $\mathrm{C}-\mathrm{G}$ & $\mathrm{C}-\mathrm{G}$ & $\mathrm{C}-\mathrm{G}$ & $\mathrm{C}-\mathrm{G}$ & $\mathrm{C}-\mathrm{G}$ & $\mathrm{C}-\mathrm{G}$ & $\mathrm{C}-\mathrm{G}$ & C-G & $\mathrm{C}-\mathrm{G}$ \\
\hline $252: 274$ & $\mathrm{~A}-\mathrm{U}$ & U-A & $\mathrm{U}-\mathrm{A}$ & $\mathrm{U}-\mathrm{A}$ & U-A & U-A & $\mathrm{U}-\mathrm{A}$ & U-A & $\mathrm{U}-\mathrm{A}$ & U-A & U-A & $\mathrm{U}-\mathrm{A}$ & U-A & $\mathrm{U}-\mathrm{A}$ & U-A \\
\hline 280 & $\mathrm{U}$ & $\mathrm{U}$ & $\mathrm{C}$ & $\mathrm{C}$ & $\mathrm{C}$ & $\mathrm{U}$ & $\mathrm{C} / \mathrm{U}$ & $\mathrm{C}$ & $\mathrm{C}$ & $\mathrm{C}$ & $\mathrm{C}$ & $\mathrm{C}$ & $\mathrm{C}$ & $\mathrm{C}$ & C \\
\hline 289:311 & $\mathrm{G}-\mathrm{U}$ & $\mathrm{U}-\mathrm{C}$ & $\mathrm{G}-\mathrm{C}$ & $\mathrm{G}-\mathrm{C}$ & $\mathrm{G}-\mathrm{C}$ & $\mathrm{G}-\mathrm{C}$ & $\mathrm{G}-\mathrm{C}$ & $\mathrm{G}-\mathrm{C}$ & $\mathrm{G}-\mathrm{C}$ & $\mathrm{G}-\mathrm{C}$ & $\mathrm{G}-\mathrm{C}$ & $\mathrm{G}-\mathrm{C}$ & $\mathrm{G}-\mathrm{C}$ & $\mathrm{G}-\mathrm{C}$ & $\mathrm{G}-\mathrm{C}$ \\
\hline $502: 543$ & $\mathrm{~A}-\mathrm{U}$ & $\mathrm{A}-\mathrm{U}$ & $\mathrm{A}-\mathrm{U}$ & $\mathrm{A}-\mathrm{U}$ & $\mathrm{A}-\mathrm{U}$ & $\mathrm{A}-\mathrm{U}$ & $\mathrm{A}-\mathrm{U}$ & $\mathrm{A}-\mathrm{U}$ & $\mathrm{A}-\mathrm{U}$ & $\mathrm{A}-\mathrm{U}$ & $\mathrm{A}-\mathrm{U}$ & $\mathrm{G}-\mathrm{C}$ & $\mathrm{A}-\mathrm{U}$ & $\mathrm{A}-\mathrm{U}$ & $\mathrm{A}-\mathrm{U}$ \\
\hline $591: 648$ & $\mathrm{U}-\mathrm{U}$ & $\mathrm{U}-\mathrm{A}$ & $\mathrm{U}-\mathrm{A}$ & $\mathrm{U}-\mathrm{A}$ & U-A & U-A & $\mathrm{U}-\mathrm{A}$ & $\mathrm{U}-\mathrm{A}$ & $\mathrm{U}-\mathrm{A}$ & $\mathrm{U}-\mathrm{A}$ & U-A & $\mathrm{U}-\mathrm{A}$ & $\mathrm{U}-\mathrm{A}$ & $\mathrm{U}-\mathrm{A}$ & $\mathrm{U}-\mathrm{A}$ \\
\hline 658 & $\mathrm{G}$ & $\mathrm{G} / \mathrm{U}$ & $\mathrm{U}$ & $\mathrm{U}$ & $\mathrm{U}$ & $\mathrm{U}$ & $\mathrm{U}$ & $\mathrm{U}$ & $\mathrm{U}$ & $\mathrm{U}$ & $\mathrm{U}$ & $\mathrm{U}$ & $\mathrm{U}$ & $\mathrm{U}$ & $\mathrm{U}$ \\
\hline $659: 746$ & U-A & $\mathrm{C}-\mathrm{G}$ & $C-G$ & $\mathrm{C}-\mathrm{G}$ & $\mathrm{C}-\mathrm{G}$ & $\mathrm{C}-\mathrm{G}$ & $\mathrm{C}-\mathrm{G}$ & $C-G$ & $\mathrm{C}-\mathrm{G}$ & $\mathrm{C}-\mathrm{G}$ & $\mathrm{C}-\mathrm{G}$ & $\mathrm{C}-\mathrm{G}$ & $\mathrm{C}-\mathrm{G}$ & C-G & $C-G$ \\
\hline 747 & $\mathrm{U}$ & $\mathrm{A} / \mathrm{C}$ & A & A & A & A & $\mathrm{A} / \mathrm{G}$ & A & A & A & A & A & A & A & A \\
\hline 811 & U & $\mathrm{C}$ & U & $\mathrm{C}$ & C & $\mathrm{U}$ & $\mathrm{C}$ & $\mathrm{C}$ & C & $\mathrm{C}$ & C & $\mathrm{C}$ & $\mathrm{C}$ & $\mathrm{C}$ & C \\
\hline 1004 & G & G & A & A & $\mathrm{A} / \mathrm{G}$ & G & A & A & A & A & A & A & A & A & A \\
\hline 1138 & G & U & $\mathrm{U}$ & U & $\mathrm{C} / \mathrm{U}$ & U & U & $\mathrm{U}$ & U & $\mathrm{U}$ & * & $\mathrm{U}$ & $\mathrm{U}$ & $\mathrm{U}$ & U \\
\hline 1159 & $\mathrm{U}$ & $\mathrm{C}$ & U & U & $\mathrm{C} / \mathrm{U}$ & U & U & $\mathrm{U}$ & U & $\mathrm{U}$ & U & $\mathrm{U}$ & $\mathrm{U}$ & $\mathrm{U}$ & U \\
\hline 1189 & U & C & $\mathrm{U}$ & $\mathrm{U}$ & $\mathrm{C} / \mathrm{U}$ & U & U & $\mathrm{U}$ & $\mathrm{U}$ & $\mathrm{U}$ & U & $\mathrm{U}$ & $\mathrm{U}$ & $\mathrm{U}$ & $\mathrm{U}$ \\
\hline 1192 & G & C & C & C & $\mathrm{C}$ & C & C & C & G & C & C & $\mathrm{C}$ & C & $\mathrm{C}$ & C \\
\hline $986: 1219$ & $\mathrm{U}-\mathrm{A}$ & $\mathrm{U}-\mathrm{U}$ & $\mathrm{U}-\mathrm{A}$ & U-A & U-A & $\mathrm{U}-\mathrm{A}$ & $\mathrm{U}-\mathrm{A}$ & $\mathrm{U}-\mathrm{A}$ & U-A & U-A & U-A & $\mathrm{U}-\mathrm{A}$ & U-A & $\mathrm{U}-\mathrm{A}$ & $\mathrm{U}-\mathrm{A}$ \\
\hline 1257 & A & $\mathrm{U}$ & $\mathrm{U}$ & * & $\mathrm{G} / \mathrm{U}$ & G & $\mathrm{G} / \mathrm{U}$ & $\mathrm{U}$ & $\mathrm{U}$ & $\mathrm{U}$ & $\mathrm{U}$ & $\mathrm{U}$ & $\mathrm{U}$ & $\mathrm{U}$ & $\mathrm{U}$ \\
\hline 1285 & A & U & $\mathrm{U}$ & U & $\mathrm{A} / \mathrm{U}$ & U & $\mathrm{U}$ & A & $\mathrm{U}$ & $\mathrm{U}$ & $\mathrm{A} / \mathrm{U}$ & $\mathrm{U}$ & $\mathrm{U}$ & G & G \\
\hline $1311: 1326$ & $\mathrm{~A}-\mathrm{U}$ & $\mathrm{A}-\mathrm{U}$ & $\mathrm{A}-\mathrm{U}$ & $\mathrm{G}-\mathrm{C}$ & $*_{-}^{*}$ & $\mathrm{~A}-\mathrm{U}$ & $\mathrm{G}-\mathrm{C}$ & $\mathrm{G}-\mathrm{C}$ & $\mathrm{G}-\mathrm{C}$ & $\mathrm{G}-\mathrm{C}$ & $\mathrm{G}-\mathrm{C}$ & $\mathrm{G}-\mathrm{C}$ & $\mathrm{G}-\mathrm{C}$ & $\mathrm{G}-\mathrm{C}$ & $\mathrm{G}-\mathrm{C}$ \\
\hline 1362 & $\mathrm{C}$ & A & A & A & $\mathrm{A} / \mathrm{G}$ & A & $\mathrm{A}$ & A & $\mathrm{A}$ & A & A & A & A & A & A \\
\hline
\end{tabular}

produced. Gelatin liquefaction, catalase and hydrolysis of Tweens 20, 40, 60 and 80 are positive. Hydrolysis of starch and cellulose, $\mathrm{H}_{2} \mathrm{~S}$ and melanin production, utilization of urea, milk coagulation, milk peptonization, nitrate reduction and oxidase are negative. The $\mathrm{pH}, \mathrm{NaCl}$ concentration and temperature ranges for growth are $\mathrm{pH} 6.0-8.0,0-13 \%$ and $10-37{ }^{\circ} \mathrm{C}$, with optimum growth at $\mathrm{pH} 7.0,3-5 \%(\mathrm{w} / \mathrm{v})$ and $28{ }^{\circ} \mathrm{C}$. Can utilize cellobiose, D-fructose, D-galactose, D-glucose, D-lactose, D-mannitol, D-mannose, D-ribose and trehalose as carbon sources, but not acetate, D-arabinose, citrate, dulcitol, inositol, maltose, raffinose, L-rhamnose, D-sorbitol, sucrose, xylitol or D-xylose.

The type strain is SCSIO $00231^{\mathrm{T}} \quad\left(=\right.$ KCTC $19433^{\mathrm{T}}$ $=$ CCTCC $\mathrm{AA}^{2} 08009^{\mathrm{T}}$ ), isolated from a marine sand sediment at a depth about $500 \mathrm{~m}$.

\section{Acknowledgements}

The authors are very grateful to Dr J. P. Euzéby for his advice on nomenclature. This research was supported by the National Basic Research Program of China (no. 2004CB719601), the National Natural Science Foundation of China (no. 30600001), the Knowledge Innovation Program of the Chinese Academy of Sciences (KZCX2-YW-216), the China National Key Program for Base Research (2005CCA04800) and the Key Project of Chinese Ministry of Education (no. 206139). W.-J.L. was supported by the Program for New Century Excellent Talents in University.

\section{References}

Al-Zarban, S. S., AI-Musallam, A. A., Abbas, I., Stackebrandt, E. \& Kroppenstedt, R. M. (2002). Saccharomonospora halophila sp. nov., a novel halophilic actinomycete isolated from marsh soil in Kuwait. Int J Syst Evol Microbiol 52, 555-558. 
Collins, M. D. \& Jones, D. (1980). Lipids in the classification and identification of coryneform bacteria containing peptidoglycans based on 2,4-diaminobutyric acid. J Appl Bacteriol 48, 459-470.

Felsenstein, J. (1985). Confidence limits on phylogenies: an approach using the bootstrap. Evolution 39, 783-791.

Fenical, W. \& Jensen, P. R. (2006). Developing a new resource for drug discovery: marine actinomycete bacteria. Nat Chem Biol 2, 666673.

Fiedler, H. P., Bruntner, C., Bull, A. T., Ward, A. C., Goodfellow, M., Potterat, O., Puder, C. \& Mihm, G. (2005). Marine actinomycetes as a source of novel secondary metabolites. Antonie van Leeuwenhoek 87, $37-42$.

Gontang, E. A., Fenical, W. \& Jensen, P. R. (2007). Phylogenetic diversity of Gram-positive bacteria cultured from marine sediments. Appl Environ Microbiol 73, 3272-3282.

Gonzalez, C., Gutierrez, C. \& Ramirez, C. (1978). Halobacterium vallismortis sp. nov., an amylolytic and carbohydrate-metabolizing, extremely halophilic bacterium. Can J Microbiol 24, 710-715.

Goodfellow, M., Kim, S. B., Minnikin, D. E., Whitehead, D., Zhou, Z. H. \& Mattinson-Rose, A. D. (2001). Amycolatopsis sacchari sp. nov., a moderately thermophilic actinomycete isolated from vegetable matter. Int J Syst Evol Microbiol 51, 187-193.

Groth, I., Rodríguez, C., Schütze, B., Schmitz, P., Leistner, E. \& Goodfellow, M. (2004). Five novel Kitasatospora species from soil: Kitasatospora arboriphila sp. nov., $K$. gansuensis sp. nov., $K$. nipponensis sp. nov., $K$. paranensis sp. nov. and $K$. terrestris sp. nov. Int J Syst Evol Microbiol 54, 2121-2129.

Jensen, P. R., Mincer, T. J., Williams, P. G. \& Fenical, W. (2005a). Marine actinomycete diversity and natural product discovery. Antonie van Leeuwenhoek 87, 43-48.

Jensen, P. R., Gontang, E., Mafnas, C., Mincer, T. J. \& Fenical, W. (2005b). Culturable marine actinomycete diversity from tropical Pacific Ocean sediments. Environ Microbiol 7, 1039-1048.

Jiang, Y., Wiese, J., Tang, S. K., Xu, L. H., Imhoff, J. F. \& Jiang, C. L. (2008). Actinomycetospora chiangmaiensis gen. nov., sp. nov., a new member of the family Pseudonocardiaceae. Int J Syst Evol Microbiol 58, 408-413.

Kelly, K. L. (1964). Inter-Society Color Council-National Bureau of Standards Color Name Charts Illustrated with Centroid Colors. Washington, DC: US Government Printing Office.

Kimura, M. (1980). A simple method for estimating evolutionary rates of base substitutions through comparative studies of nucleotide sequences. J Mol Evol 16, 111-120.

Kimura, M. (1983). The Neutral Theory of Molecular Evolution. Cambridge: Cambridge University Press.

Korn-Wendisch, F., Kempf, A., Grund, E., Kroppenstedt, R. M. \& Kutzner, H. J. (1989). Transfer of Faenia rectivirgula Kurup and Agre 1983 to the genus Saccharopolyspora Lacey and Goodfellow 1975, elevation of Saccharopolyspora hirsuta subsp. taberi Labeda 1987 to species level, and emended description of the genus Saccharopolyspora. Int J Syst Bacteriol 39, 430-441.

Korn-Wendisch, F., Rainey, F., Kroppenstedt, R. M., Kempf, A., Majazza, A., Kutzner, H. J. \& Stackebrandt, E. (1995). Thermocrispum gen. nov., a new genus of the order Actinomycetales, and description of Thermocrispum municipale sp. nov. and Thermocrispum agreste sp. nov. Int J Syst Bacteriol 45, 67-77.

Kroppenstedt, R. M. (1982). Separation of bacterial menaquinones by HPLC using reverse phase (RP18) and a silver loaded ion exchanger as stationary phases. J Liq Chromatogr 5, 2359-2367.

Kroppenstedt, R. M., Korn-Wendisch, F., Fowler, V. J. \& Stackebrandt, E. (1981). Biochemical and molecular genetic evidence for a transfer of Actinoplanes armeniacus into the family Streptomycetaceae. Zentralbl Bakteriol Mikrobiol Hyg 1 Abt Orig C 2, 254-262.

Laatsch, H. (2006). Marine bacterial metabolites. In Frontiers in Marine Biotechnology, pp. 225-288. Edited by P. Proksch \& W. E. G. Müller. Wymondham, UK: Horizon Bioscience.

Lam, K. S. (2006). Discovery of novel metabolites from marine actinomycetes. Curr Opin Microbiol 9, 245-251.

Lechevalier, M. P., De Bièvre, C. \& Lechevalier, H. A. (1977). Chemotaxonomy of aerobic actinomycetes: phospholipid composition. Biochem Syst Ecol 5, 249-260.

Lee, S. D., Kim, E. S., Kang, S. O. \& Hah, Y. C. (2002). Pseudonocardia spinosispora sp. nov., isolated from Korean soil. Int J Syst Evol Microbiol 52, 1603-1608.

Li, W.-J., Xu, P., Schumann, P., Zhang, Y.-Q., Pukall, R., Xu, L.-H., Stackebrandt, E. \& Jiang, C.-L. (2007). Georgenia ruanii sp. nov., a novel actinobacterium isolated from forest soil in Yunnan (China), and emended description of the genus Georgenia. Int J Syst Evol Microbiol 57, 1424-1428.

Majumdar, S., Prabhagaran, S. R., Shivaji, S. \& Lal, R. (2006). Reclassification of Amycolatopsis orientalis DSM 43387 as Amycolatopsis benzoatilytica sp. nov. Int J Syst Evol Microbiol 56, 199-204.

Maldonado, L. A., Stach, J. E. M., Pathom-aree, W., Ward, A. C., Bull, A. T. \& Goodfellow, M. (2005). Diversity of cultivable actinobacteria in geographically widespread marine sediments. Antonie van Leeuwenhoek 87, 11-18.

Mertz, F. P. \& Yao, R. C. (1993). Amycolatopsis alba sp. nov., isolated from soil. Int J Syst Bacteriol 43, 715-720.

Mesbah, M., Premachandran, U. \& Whitman, W. B. (1989). Precise measurement of the $\mathrm{G}+\mathrm{C}$ content of deoxyribonucleic acid by highperformance liquid chromatography. Int J Syst Bacteriol 39, 159-167.

Minnikin, D. E., Collins, M. D. \& Goodfellow, M. (1979). Fatty acid and polar lipid composition in the classification of Cellulomonas, Oerskovia and related taxa. J Appl Bacteriol 47, 87-95.

Minnikin, D. E., O'Donnell, A. G., Goodfellow, M., Alderson, G., Athalye, M., Schaal, A. \& Parlett, J. H. (1984). An integrated procedure for the extraction of bacterial isoprenoid quinones and polar lipids. J Microbiol Methods 2, 233-241.

Saitou, N. \& Nei, M. (1987). The neighbor-joining method: a new method for reconstructing phylogenetic trees. Mol Biol Evol 4, 406-425.

Sasser, M. (1990). Identification of bacteria by gas chromatography of cellular fatty acids. USFCC Newsl 20, 16.

Schleifer, K. H. (1985). Analysis of the chemical composition and primary structure of murein. Methods Microbiol 18, 123-156.

Schleifer, K. H. \& Kandler, O. (1972). Peptidoglycan types of bacterial cell walls and their taxonomic implications. Bacteriol Rev 36, 407-477.

Shirling, E. B. \& Gottlieb, D. (1966). Methods for characterization of Streptomyces species. Int J Syst Bacteriol 16, 313-340.

Smibert, R. M. \& Krieg, N. R. (1981). General characterization. In Manual of Methods for General Bacteriology, pp. 409-443. Edited by P. Gerhardt, R. G. E. Murray, R. N. Costilow, E. W. Nester, W. A. Wood, N. R. Krieg \& G. B. Phillips. Washington, DC: American Society for Microbiology.

Stach, J. E. M., Maldonado, L. A., Ward, A. C., Goodfellow, M. \& Bull, A. T. (2003). New primers for the class Actinobacteria: application to marine and terrestrial environments. Environ Microbiol 5, 828-841.

Stackebrandt, E., Rainey, F. A. \& Ward-Rainey, N. L. (1997). Proposal for a new hierarchic classification system, Actinobacteria classis nov. Int J Syst Bacteriol 47, 479-491. 
Staneck, J. L. \& Roberts, G. D. (1974). Simplified approach to identification of aerobic actinomycetes by thin-layer chromatography. Appl Microbiol 28, 226-231.

Tamura, K., Dudley, J., Nei, M. \& Kumar, S. (2007). MEGA4: molecular evolutionary genetics analysis (MEGA) software version 4.0. Mol Biol Evol 24, 1596-1599.

Thompson, J. D., Gibson, T. J., Plewniak, F., Jeanmougin, F. \& Higgins, D. G. (1997). The CLUSTAL_X windows interface: flexible strategies for multiple sequence alignment aided by quality analysis tools. Nucleic Acids Res 25, 4876-4882.
Tomita, K., Hoshino, Y. \& Miyaki, T. (1993). Kibdelosporangium albatum sp. nov., producer of the antiviral antibiotics cycloviracins. Int J Syst Bacteriol 43, 297-301.

Waksman, S. A. (1961). The Actinomycetes, vol. II, Classification, Identification and Description of Genera and Species. Baltimore: Williams \& Wilkins.

Xu, P., Li, W. J., Tang, S. K., Zhang, Y. Q., Chen, G. Z., Chen, H. H., Xu, L. H. \& Jiang, C. L. (2005). Naxibacter alkalitolerans gen. nov., sp. nov., a novel member of the family Oxalobacteraceae isolated from China. Int J Syst Evol Microbiol 55, 1149-1153. 